\title{
Nivel de conocimientos de los adolescentes sobre el Virus de Inmunodeficiencia Humana/ Síndrome de Inmunodeficiencia Adquirida.
}

\author{
Level of knowledge among adolescents on \\ the Human Immunodeficiency Virus/Acquired \\ Immunodeficiency Syndrome.
}

PSS. Antia Jiménez Ruíz•, PSS. Fabiola Jiménez García•, MCE. Inés Tenahua Quitl••,

MCE. Ignacio Grajales Alonso.•.

\section{Resumen}

El Programa Conjunto de las Naciones Unidas informó que 33.3 millones de personas en el mundo vivían con el VIH a finales del 2009. En México existen 200,000 personas con el VIH/SIDA. En el sureste mexicano, Oaxaca ocupa el cuarto lugar de casos de SIDA principalmente en los adolescentes. La disciplina de enfermería puede abordar este problema a través de la promoción y prevención de la enfermedad en los adolescentes que residen en zonas rurales. Objetivo. Identificar el nivel de conocimientos que tienen los adolescentes hijos de padres migrantes acerca del VIH/SIDA de una comunidad rural oaxaqueña. Metodología. El estudio fue transversal, descriptivo. La muestra fue de 37 adolescentes ambos sexos hijos de padres migrantes. La ética del estudio consideró el Reglamento de la Ley General de Salud. Resultados. El 91.2\% de la muestra presentó conocimientos regulares, el $2.9 \%$ conocimientos adecuados y el $5.9 \%$ no adecuados. Discusión y conclusión. Los resultados coinciden con otras investigaciones, sin embargo la mayor parte de éstas se han realizado en un contexto urbano, lo que ha limitado la prevención del VIH/SIDA en zonas rurales. El estudio, permitió evidenciar problemas que pueden ser abordados, por la disciplina de enfermería.

- Pasantes de la Licenciatura en Enfermería de la Universidad de la Sierra Sur.

•Maestros en Ciencias de Enfermería. Profesores-Investigadores de tiempo completo de la Universidad de la Sierra Sur.

Correspondencia: inestq@hotmail.com; cel 0442224 476234; Fax 019515724100.

FECHA DE RECIBIDO: 15 DE FEBRERO DE 2011

Fecha de ENVIado: 30 DE MARZo DE 2011

FeCha DE ACEPTADo: 17 DE MAYO DE 2011 


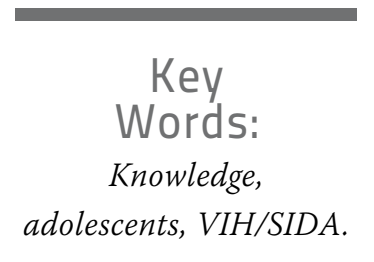

Key

Words:

adolescents, VIH/SIDA.
AbSTRACT

The United Nation Joint Program of the United Nations Organization to inform that 33.3 millions of persons in the world lived with VIH to the end of 2009. In México exist 200,000 persons with VIH/SIDA. Oaxaca hold the fourth position of case of SIDA in the southeast in adolescents. The discipline of nursing can broach this problem through it promotion and prevention of the disease in the adolescents that to lie in place zone rurals. Objective. To identify the level of knowledge that to have the adolescents children's of parents migrants about the one VIHISIDA of a community rural of Oaxaca. Methodology. The study were descriptive transverse. The simple were of 37 adolescents both sexs. Children's of parents migrants. The ethic of study to considers the Rules Health General Law of México. Results. The $91.2 \%$ of sample to present knowledge regulars; the $2.9 \%$ knowledge good and the $5.9 \%$ knowledge bads. Discussion and conclusion. The results to coincide with others researchs. However the majority part of these to them the used in a context urban, this to limited prevention of VIHISIDA in zones rurals. The adolescents had Knowledge regulars and the study to permit to demonstrate problems that can be broach by the discipline of nursing.

\section{INTRODUCCIÓN}

El Programa Conjunto de las Naciones Unidas sobre el Virus de Inmunodeficiencia Humana/ Síndrome de Inmunodeficiencia Adquirida (VIH/SIDA) informó que $33.3 \mathrm{mi}$ llones de personas en el mundo vivían con el VIH a finales del 2009, de las cuales 2.7 millones contrajeron la infección y 2 millones fallecieron por causas relacionadas con este padecimiento, por lo que se ha considerado como una prioridad sanitaria en el mundo ${ }^{1,2}$ Esta epidemia, ha tenido un efecto particularmente devastador en la vida de las mujeres ya que representan el $66 \%$ de las infecciones entre los jóvenes en el mundo. Siendo que éstos representan el $40 \%$ de todas las nuevas infecciones por el VIH en personas entre los 15 y 49 años se considera que cerca de 3,000 jóvenes se infectan con el viH cada día. ${ }^{3}$

El Caribe ocupa el segundo lugar más alto de prevalencia del VIH, especialmente entre mujeres adolescentes y jóvenes, quienes tien- den a presentar tasas significativamente más altas que los varones de la misma edad. ${ }^{2}$ Por lo tanto para el año 2009 alrededor de 240,000 personas vivían con el VIH, de las cuales, 17,000 se infectaron con el virus y 12,000 fallecieron a causa del SIDA. ${ }^{1}$ En América Latina el total estimado de nuevas infecciones fue de 170,000 y, en consecuencia, el número de personas que viven con el VIH ascendió a 2 millones. De ahí que, aproximadamente 77,000 personas fallecieron a causa del SIDA en este lugar. ${ }^{2}$ En estas regiones, la epidemia está bien consolidada y asume muchas facetas, dada la diversidad en los patrones demográficos y de desarrollo de los países de la región, por lo cual, existe el peligro de que aumenten de forma rápida más casos. ${ }^{4}$ Derivado de lo anterior se considera importantes el conocimiento y la información ya que son las primeras líneas de defensa para evitar contraer el VIH/SIDA en los adolescentes. Sin embargo el acceso a la educación sobre estos padecimientos está lejos de ser universal siendo que en estas zonas geográficas sólo el 4\% de los jóvenes no escolarizados y el $38 \%$ de los escolarizados tienen acceso a la educación sobre el tema, aunado a ello, existen respuestas nacionales poco eficaces reflejadas en un acceso inadecuado de los servicios de prevención y tratamiento de estos padecimientos, lo que ha contribuido a la propagación acelerada del VIH/SIDA en las Américas. ${ }^{5}$ En México, al igual que en el resto de los países del mundo, se ha convertido en un problema prioritario de salud pública complejo con múltiples repercusiones psicológicas, sociales, éticas, económicas y políticas las que incluso rebasan el ámbito de la salud.

De acuerdo al Centro Nacional para la Prevención y Control del VIH/SIDA, durante 2006 se identificaron 4,944 casos de muerte a consecuencia del virus, lo que representa una tasa de 4.7 por cada 100 mil habitantes. De igual mane- 
ra el mayor porcentaje de personas infectadas es el grupo de 30 a 44 años, en un $49.5 \%$ hombres y $42.0 \%$ mujeres, seguido de la población de 15 a 19 años con un $29.5 \%$ hombres y 33.6\% mujeres. También es alarmante que cerca de 3 mil personas menores de 15 años, vivan con la infección. ${ }^{6}$ Particularmente el estado de Oaxaca tiene registrados 4 mil 784 casos acumulados de SIDA, con esta cifra la entidad se ubica en el cuarto lugar dentro de los estados que conforman la región Sur-Sureste y el décimo primer lugar a nivel nacional. La incidencia de personas infectadas con SIDA en el 2010 fue de 134.8 casos por cada 100 mil habitantes y la tasa de mortalidad fue 5.6 por cada $100 \mathrm{mil}$ habitantes en el 2008. ${ }^{7}$ Es necesario señalar que este estado posee un gran número de municipios con un precario índice de desarrollo sustentable y de alta marginación, caracterizados por escasas oportunidades en educación y pobreza extrema, condiciones que han influido para que los habitantes de zonas rurales e indígenas opten por la migración a otros países principalmente a los Estados Unidos, razón por la cual, se estima que la cuarta parte de los casos de VIH/ SIDA en el país ocurren en personas que han pasado períodos largos de estancia en los Estados Unidos de Norte América para posteriormente regresar a nuestro país. ${ }^{8}$

Por otra parte, el sector salud mexicano todavía cuenta con recursos materiales y humanos insuficientes y además se presentan problemas de distribución entre entidades federativas, instituciones y poblaciones. ${ }^{9}$ Aunado a esto existen aún tensiones importantes en el desarrollo de los adolescentes y los jóvenes, particularmente en el aspecto de la desigualdad social que persiste entre la población de México y genera una alta diversidad de situaciones que limitan las estructuras de oportunidades en las que se desenvuelven los jóvenes, generando mayor vulnerabilidad entre ellos, en particular entre los adolescentes, los pobladores de entidades con menores niveles de desarrollo, quienes residen en contextos rurales, los hablantes de lengua indígena $y$, aún en nuestros días, las mujeres. ${ }^{10}$ Ante la evidencia, existe la urgencia de abordar el tema del VIH/SIDA por medio de la educación para la salud como parte de la promoción y prevención para garantizar la adquisición o reafirmación de conocimientos sobre el tema, ya que la mayoría de los jóvenes, no tienen suficiente acceso a programas de salud sexual y reproductiva que les proporcionen información, habilidades, servicios, productos básicos y el apoyo social que requieren para prevenir el VIH, y con ello, la

\begin{tabular}{|} 
En México, al igual \\
que en el resto de los \\
países del mundo, \\
se ha convertido \\
en un problema \\
prioritario de salud \\
pública complejo con \\
múltiples repercusiones \\
psicológicas, sociales, \\
éticas, económicas \\
y políticas las que \\
incluso rebasan \\
el ámbito de la salud.
\end{tabular}

reducción en la propagación de este indicador de salud particularmente en quienes viven en zonas rurales e indígenas.

En México residen 20.2 millones de personas entre 15 y 24 años de edad los cuales representan cerca de la quinta parte de la población total (108.4 millones). De ellos, la mitad (10.4 millones) son adolescentes y el resto son adultos jóvenes (9.8 millones) ${ }^{10}$ los cuales debido a sus atributos físicos, psicológicos y sociales contribuyen a que corran un alto riesgo de infectarse con el VIH por muchas razones, entre las que se incluyen una combinación de factores biológicos, las diferencias por razón de sexo, inicio sexual precoz, falta de acceso a información educación y servicios sobre el VIH, desempoderamiento a menudo abusivo de las condiciones sociales, culturales y económicas, coacción sexual, violencia de género, el uso de drogas inyectables y la venta de sexo, entre otras. ${ }^{3}$ Esto obliga a los jóvenes hombres y mujeres a tomar decisiones difíciles y riesgosas y a hacerlos particularmente vulnerables al VIH y otras infecciones de transmisión sexual (ITS). ${ }^{11}$

Algunos estudios han abordado el tema de interés mostrando que el nivel de conocimientos de los adolescentes sobre el VIH/SIDA es adecuado $^{12,13,14}$ sin embargo, otras investigaciones han evidenciado que los conocimientos acerca de estos padecimientos son regulares, insuficientes y escasos, como lo mencionan Macchi, Benítez, Corvalán, Nuñez y Ortigoza. ${ }^{5}$ Respecto de la fuente de información en relación al VIH/SIDA se da con mayor auge en la escuela. En cuanto al inicio de relaciones sexuales la edad 
promedio es de 14.6 años, $72 \%$ de los varones refieren antes de los 15 años. Un 55\% refieren utilizar preservativo en relaciones ocasionales. Esto es similar a lo que refieren Navarro y Vargas ${ }^{15}$ quienes muestran que los adolescentes de 14 a 19 años no tienen suficientes conocimientos sobre esta enfermedad. Esto se evidenció ya que los adolescentes desconocen las formas de prevención y transmisión del VIH/ SIDA, siendo que el $33 \%$ ha iniciado su vida sexual activa y de éstos un gran porcentaje presenta prácticas de riesgo como consumir alcohol, sexo con desconocidos y no usar preservativo en distintas ocasiones. Ellos concluyen que el educar en este tema proporcionará una modificación sobre sus conductas de riesgo. Otros autores ${ }^{16}$ en su investigación determinaron que el nivel de conocimientos de los jóvenes es regular, así mismo, reportaron que el inicio de relaciones sexuales fue a los 14.1 años en los varones y 15.4 años en las mujeres. Uno de cada tres varones refirió haber tenido relaciones sexuales a diferencia de una de cada doce mujeres; de éstos 30\% manifestó no haber usado preservativos en sus relaciones sexuales sin diferencias según sexo. También mencionan que la información sobre VIH/SIDA es recibida principalmente de folletos (69.4\%), televisión (64.2\%) y de sus profesores $(62.2 \%)$. Concluyeron que el inicio precoz de relaciones sexuales y el uso limitado de preservativos muestran un riesgo de contraer la infección a temprana edad. Este estado del arte muestra estudios en los cuales se ha abordado el tema de investigación principalmente en zonas urbanas por lo que la presente investigación se centró en una población rural, por la cual el objetivo del estudio fue: Identificar el grado de conocimientos acerca del $\mathrm{VIH} / \mathrm{SIDA}$ que tienen adolescentes de 12 a 18 años hijos de padres migrantes de la comunidad rural de San Francisco Coatlán, Oaxaca.

\section{Metodología}

El estudio fue de tipo descriptivo, transversal. La población de interés la constituyen 326 adolescentes de 12 a 18 años, de ambos sexos de la comunidad de San Francisco Coatlán, de los cuales 82 son hijos de padres migrantes.

Esta comunidad se caracteriza por poseer un alto índice de actividad migratoria, hacinamiento en hogares, bajo ingreso económico, fauna nociva y escasez de servicios básicos sanitarios, de educación y recreación. A su vez, se rigen por creencias y costumbres; cuenta con casas dispersas de acuerdo a su ubicación geográfica y su vía de acceso es escasa.

El muestreo fue de tipo aleatorio simple procediendo de un marco muestral. El tamaño de la muestra se calculó a través de proporción de poblaciones finitas por lo cual se estableció un nivel de confianza de $90 \%$ y con un margen de error de $0.1 .{ }^{17}$ La muestra estuvo conformada por 37 adolescentes.

Para la recolección de la información se utilizó el instrumento de "Trabajo para el estudio de las enfermedades de transmisión sexual y VIH/SIDA en adolescentes" del Instituto Nacional de Higiene, Epidemiología y Microbiología de Cuba. ${ }^{18}$ El cuestionario está constituido por 42 preguntas subdivididas en 5 apartados: el primero explora datos sociodemográficos; el segundo aborda los aspectos re- lacionados con los conocimientos sobre las ITS y VIH/SIDA; el tercero se refiere a las actitudes y el comportamiento sexual; el cuarto indaga sobre antecedentes de ITS y manifestaciones clínicas relacionadas; y el quinto toca posibles factores relacionados con las ITS/VIH/ SIDA. Para la medición del nivel de conocimiento se utilizó una escala de intervalo en donde se incluyeron 10 reactivos a los cuales se les asignó un puntaje, y se clasificaron de la siguiente manera: de 0 a 6 "Nivel de conocimientos deficiente"; 7 a 13 "Nivel de conocimientos regulares"; y 14 a 20 "Nivel de conocimientos aceptables". El instrumento ha sido aplicado en diversas investigaciones por los mismos autores, sin embargo no reportan coeficientes de confiabilidad. De esta manera, en la presente investigación, se aplicó el coeficiente Alpha de Cronbach para determinar la confiabilidad del instrumento, el cual fue de .84. El estudio se llevó a cabo de acuerdo con al Reglamento de la Ley General de Salud en Materia de Investigación para la Salud con base en el título segundo, capítulo uno, por ello se respetó la dignidad, la protección de sus derechos y bienestar de los participantes llevándose a cabo los principios científicos y éticos; se contó con el consentimiento informado del participante y un testigo por escrito y verbal. Con ello se protegió la privacidad y confidencialidad de la información proporcionada. Así mismo, el investigador principal suspenderá la investigación de inmediato cuando el sujeto de investigación así lo manifieste. El estudio fue considerado de riesgo mínimo (Art. 13, Art. 14 fracción (I, V, VI), art. 16, art. 17 fracción 
(I) y $18^{19}$. Para el análisis de los datos se utilizó el paquete estadístico Epi-info. Este programa permitió la obtención de estadística descriptiva (porcentajes, moda, mediana, media) para la presentación de los datos y la interpretación de los resultados.

\section{Resultados}

La muestra fue de 37 adolescentes, el 59.5 \% correspondió al sexo femenino y el $40.5 \%$ al sexo masculino. La edad promedio fue de 14 años. Con respecto a la escolaridad el 59.5\% cursa la educación secundaria, un $27 \%$ no estudia, el $8.1 \%$ corresponde a preuniversitarios y un 5.4\% cursa la primaria. El 70\% vive con ambos padres y el $2.7 \%$ con su pareja. En cuanto al nivel de conocimientos del VIH/SIDA en los adolescentes el $91.2 \%$ presentó conocimientos regulares (Gráfica 1). Los datos estadísticos que determinaron el nivel de conocimientos fueron los siguientes: el $83.8 \%$ de los adolescentes tienen una definición sólida acerca del VIH/SIDA y de las vías de transmisión, en tanto que el $43.8 \%$ conoce los factores que favorecen el contagio de la infección, y sólo un $29.7 \%$ asegura saber las consecuencias de estas alteraciones en su salud. Cabe mencionar que un $78.6 \%$ de los adolescentes refieren que en la escuela se les ha proporcionado información acerca del VIH/SIDA, y que solo el $21.4 \%$ manifiesta haber recibido información por parte de un médico.

En cuanto a la calidad de la información recibida, el $56.7 \%$ respondió que fue buena, el $26.7 \%$ comenta que fue regular y solo el $6.7 \%$ refiere que fue deficiente. Un 48.6\% de los adolescentes cree que el SIDA es una enfermedad vergon-

\section{Gráfica 1. Nivel de conocimientos de los adolescentes sobre el VIH/SIDA.}

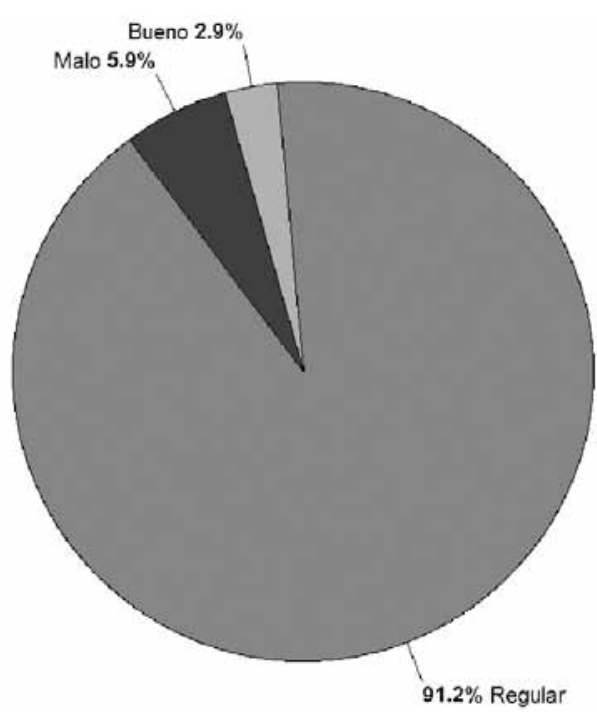

Gráfica 2. Creencias de los adolescentes sobre el VIH/SIDA.

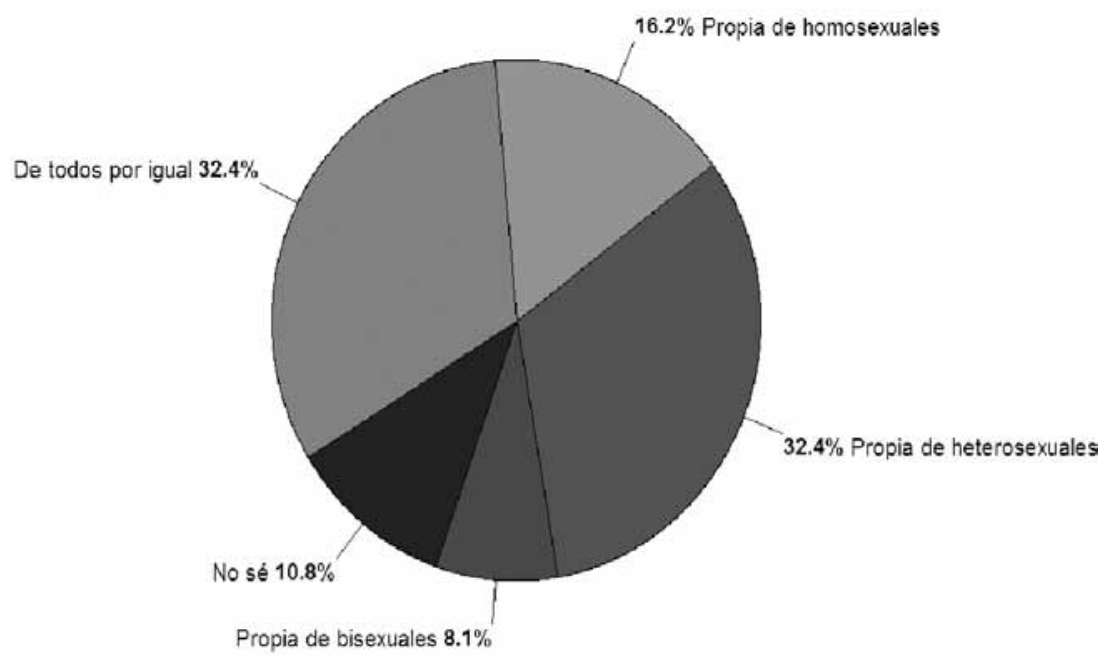




\section{Gráfica 3. Decisión de los adolescentes de tener una sola pareja.}

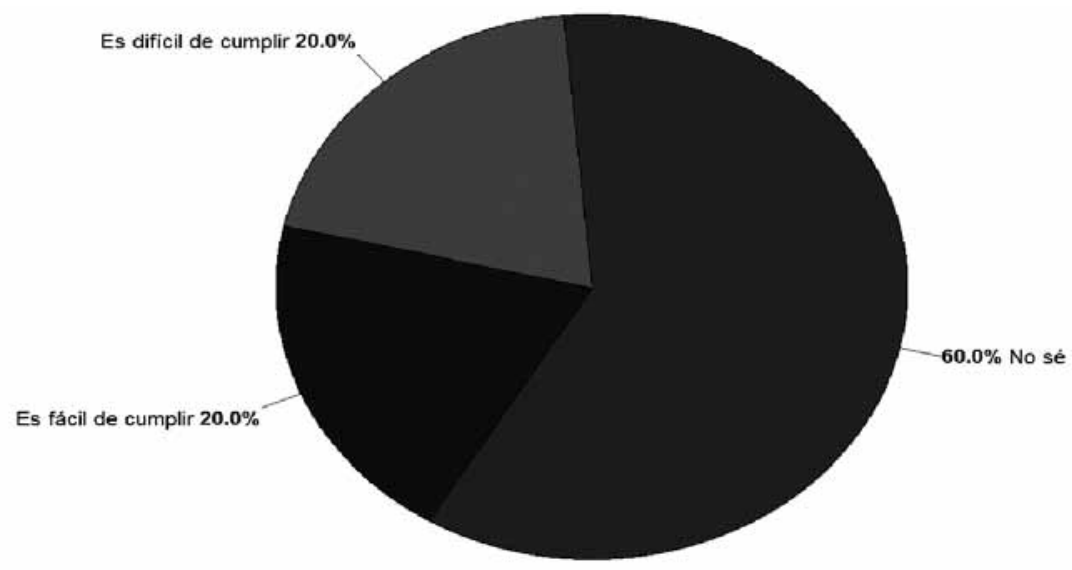

zosa e inmoral, el $27 \%$ manifestó que es un padecimiento del mal ambiente y solo el $24.3 \%$ considera que es como otra cualquiera.

Con respecto a las creencias del $\mathrm{VIH} / \mathrm{siDA}$, el $32.4 \%$ respondió que es una enfermedad propia de heterosexuales (Gráfica 2). En lo que concierne a las actitudes y comportamiento sexual de los adolescentes frente al VIH/SIDA se encontró que el $81.1 \%$ no ha tenido relaciones sexuales, sin embargo, el $13.5 \%$ no respondió y solo un $5.4 \%$ si refirió haber tenido relaciones, y la edad de inicio fue de 16 y 15 años, ambas con un $50 \%$.

Así mismo, el 60\% manifiesta no tener otra pareja sexual, el $40 \%$ no respondió y el 100\% menciona solo haber tenido una sola pareja sexual. Cabe señalar que la decisión de tener una sola pareja se les dificulta a los adolescentes (Gráfica 3). En cuanto al conocimiento del uso del preservativo para prevenir el contagio, el $45.9 \%$ considera que no protege, el $29.7 \%$ respondió que no sabe y el $21.6 \%$ considera que no siempre protege, en tanto el $2.7 \%$ respondió que si protege, bajo este mismo tema, el $54.4 \%$ si ha recibido explicación sobre el uso del preservativo, $43.2 \%$ no y $5.4 \%$ no respondió.

\section{Discusıón}

El estudio tuvo como objetivo identificar el nivel de conocimientos que tienen los adolescentes hijos de padres migrantes acerca del VIH/ SIDA. Los resultados obtenidos de la investigación muestran que los adolescentes tienen conocimientos regulares, esto es similar al estudio realizado por Catácora y Villanueva. ${ }^{16}$ Una posible explicación sería que a pesar de que se ha otorgado información a los adolescentes que habitan en las zonas indígenas o rurales, ésta no ha sido suficiente o quizá sea la falta de recursos sanitarios para estos lugares en donde una de sus características principales es el grado de marginación.

Por otra parte Macchi, Benítez, Corvalán, Nuñez y Ortigoza, ${ }^{5}$ Navarro y Vargas ${ }^{15}$ refieren que los jóvenes poseen un nivel de cono- cimientos bajos, inconsistentes e insuficientes sobre el VIH/SIDA, por lo tanto consideran que ellos tienen una situación de riesgo para contraer el VIH/SIDA. A su vez Navarro y Vargas consideran que el educar en esta enfermedad proporcionará una modificación sobre sus conductas de riesgo. Estos resultados coincidieron con el Programa Conjunto de las Naciones Unidas en donde los jóvenes corren un alto riesgo de infectarse por la falta de acceso a información, educación y servicios sobre el VIH. ${ }^{2}$ Esto difiere con lo encontrado en el presente trabajo, lo cual pudiera explicarse si partimos de considerar a la adolescencia como una etapa de cambio que se caracteriza por poseer atributos físicos, psicológicos, sociales y económicos que al canalizarlos de manera negativa pueden generar prácticas inseguras para la prevención, lo cual convierte a los adolescentes en un grupo de riesgo para contraer el VIH/SIDA (a pesar de que existe un mayor acceso de la educación en la zona urbana). También lo obtenido en esta investigación contrasta con otros autores $^{12,13,14}$ quienes reportaron un nivel de conocimientos adecuados, esto puede atribuirse o estar relacionado con el contexto de la educación formal, siendo las muestras de estos estudios estuvieron conformadas por estudiantes de distintos grados y la información respecto al VIH/SIDA pudiera estar más consolidada en los docentes.

Respecto al conocimiento que han recibido los adolescentes acerca del VIH/SIDA, éstos refieren haberlo obtenido dentro de la formación escolar y mínimamente por parte del equipo de salud. Este hallazgo llama la atención, pues se 
esperaría que fuese a la inversa ya que los profesionales de la salud en esencia son los responsables directos de brindar este tipo de información, como parte de la promoción y prevención de la salud, dirigida no solo a los adolescentes sino a la población en general. Quizá por tratarse de zonas rurales consideradas socialmente como regiones con un bajo índice de desarrollo, la lógica lleva a pensar que existe una carencia de servicios sanitarios idóneos, de infraestructura adecuada, falta de mobiliario y equipo y, sobre todo, de recursos humanos por lo que no se puede garantizar que se satisfagan de manera eficaz las necesidades de salud (e información sobre su cuidado, en este caso) que presenta la población indígena o rural. Por consiguiente, se hace difícil el proveer de suficiente información por parte del equipo de salud a los habitantes de estos lugares y quizá por ello no se ha logrado impactar en este indicador que afecta a un gran número de adolescentes en México.

Derivado de lo anterior pareciera ser obvio que al carecer de información los adolescentes reflejan creencias y actitudes erróneas acerca del VIH/sidA, lo que los puede exponer a un inevitable contagio de estos padecimientos. En la presente investigación se encontró que los adolescentes conocen las formas de transmisión de estos padecimientos, sin embargo, esto no se traduce a que el adolescente lleve a cabo conductas sexuales seguras para prevenir el contagio, ya que en lo respecta a la protección que ofrece el preservativo, el $45 \%$ de la muestra refirió que no siempre protege, este resultado concuerda Navarro $y \operatorname{Vargas}^{15}$ quienes mencionan que

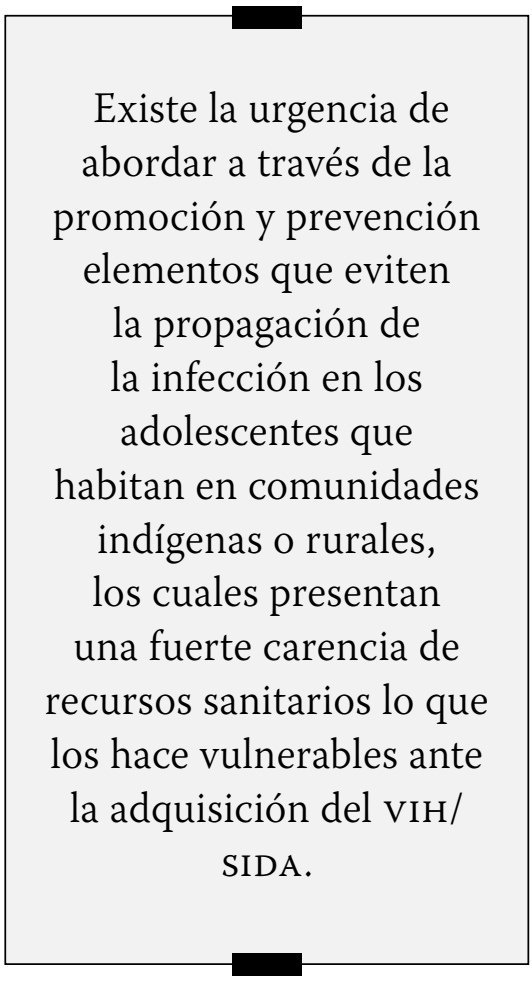

los adolescentes no tienen conocimientos de que el preservativo es una forma de prevenir el contagio, así mismo, no saben cómo usarlo.

La anterior discusión muestra la urgencia de abordar a través de la promoción y prevención elementos que eviten la propagación de la infección en los adolescentes que habitan en comunidades indígenas o rurales, los cuales presentan una fuerte carencia de recursos sanitarios lo que los hace vulnerables ante la adquisición del VIH/SIDA. Por otra parte, el número de casos de este padecimiento siguen en aumento y México, es una de los países de la región Latinoamericana que más ha sentido el impacto de este indicador de salud, por lo tanto, los equipos sanitario y gubernamental deben de crear de manera conjunta, estrategias de salud dirigidas a prevenir el contagio del VIH/SIDA y evitar con ello gastos innecesarios para el sistema nacional de salud, político y familiar.

\section{Conclusiones}

Este estudio mostró que los adolescentes hijos de padres migrantes tienen un nivel de conocimientos regulares acerca del VIH/SIDA, por lo que el conocimiento se encuentra de manera poco sólida en este grupo etario. Se evidencian algunos factores que han sido parcialmente atendidos por parte del sistema gubernamental y del equipo de salud en México. Sin embargo se requieren mayores esfuerzos sobre todo en las poblaciones de alta marginación.

Quedan otros temas de interés para ser indagados en un futuro mediato por parte de la disciplina de enfermería que contribuyan en la solución de estas problemáticas de salud que ponen en riesgo a los adolescentes y de la población indígena o rural ante el posible contagio del VIH/SIDA. Es recomendable seguir desarrollando el instrumento utilizado que permita tener una mayor clarificación en cuanto a las escalas de medición para las variables. Metodológicamente será necesario también ampliar el tamaño de la muestra o hacia otras poblaciones para poder tener un marco de análisis del comportamiento de la población de esta región de nuestro país respecto a este tema.

\section{REFERENCIAS BILIOGRÁFICAS}

1 Joint United Nations Programme on HIV/AIDS. (UNAIDS). Global Report: UNAIDS report on the global AIDS epidemia. 2010; Disponible en: http://bit.ly/vlMwGE [consulta 20 de enero de 2011]

2 Programa Conjunto de las Naciones Unidas sobre el VIH/SIDA. Situación de la epidemia de sida 2009. Disponible en: http://bit.ly/dXL7Z8 [consulta 21 de enero de 2011]. 
3 Programa Conjunto de las Naciones Unidas sobre el VIH/SIDA. Podemos empoderar a los jóvenes para que se protejan del VIH 2010. Disponible en: http://bit.ly/sJVBNu [consulta 20 de enero de 2011]

4 Estrada JH. Modelos de Prevención en la Lucha contra el VIH/SIDA. Acta bioeth. [Internet]. 2006 Ene 12(1): [91-100]. Disponible en: http://www.scielo.cl/scielo.php?p $\mathrm{id}=$ S1726569X2006000100013\&s cript=sci_arttext [consulta 20 de enero de 2011].

5 Macchi ML, Benítez LS, Corvalán A, Nuñez C, Ortigoza D. Conocimientos, actitudes y prácticas acerca del VIH/SIDA en jóvenes de nivel medio de Educación, del área metropolitana. Rev. Chil. Pediatr. 2008 79(2): [206-17]. [Internet] Disponible en: http://bit.ly/ tnHRGZ [consulta 21 de enero de 2011]

6 Instituto Nacional de Estadística y Geografía. Estadísticas a propósito del día mundial de la lucha contra el Sida. Datos Nacionales. Disponible en: http://bit.ly/u1cmqm [consulta 21 de enero de 2011].

7 Instituto Nacional de Estadística y Geografía. Estadísticas a propósito del día mundial de la lucha contra el sida. Datos de Oaxaca. Disponible en: www.inegi.org.mx/ inegi/contenidos/espanol/prensa/.../2010/sida20.doc [consulta 20 de mayo de 2011]

8 Center for AIDS Prevention Studies AIDS Research Institute. ¿Cuales son las necesidades de prevención de inmigrantes mexicanos/ as en EE.UU? 2003. Disponible en: http://bit.ly/v15BvH [consulta 22 de enero de 2011]

9 Secretaría de Salud. Programa Nacional de Salud 2007-2012 Por un México sano: construyendo alian- zas para una mejor salud. 2007. Disponible en: http://bit.ly/szxJ1x [consulta 23 de enero 2011];

10 Secretaría General del Consejo Nacional de la Población (CONAPO). La Situación actual de los jóvenes en México. Disponible en: http:// bit.ly/tcTtFG [consulta 20 enero de 2011].

11 Kiragu K. Los jóvenes y el VIH sida, ¿podemos evitar una catástrofe? Population Reports. Disponible en: http://bit.ly/sfv9mZ [consulta 20 enero de 2011]

12 Cortés A, García R, Monterrey P, Fuentes J, Pérez D. Sida, adolescencia y riesgos. Rev Cubana Med Gen Integr. 2000;16(3): [253-60]. [Internet] Disponible en: http:// bit.ly/tV1H2I [consulta 30 de enero de 2011]

13 Caballero H, Villaseñor S. Conocimientos sobre VIH/SIDA en adolescentes urbanos: consenso cultural de dudas e incertidumbres. Salud Pública Mex 2003; 45 (Supl. 1): [108-114]. [Internet] Disponible en: http://bit.ly/umXPmC [consulta 01 de febrero de 2011]

14 Flores BF, Castañeda HH, Quintero VL, González QN, Garza HR, Mata RM. Conocimientos de Adolescentes en la Promoción de la Salud para Prevenir Enfermedades de Transmisión Sexual. Desarrollo Cientif Enferm. 2008; 16(4): 161-4.

15 Navarro L, Vargas M. Conocimientos, actitudes y prácticas sobre SIDA en adolescentes escolarizados. Revistas científicas de América Latina y el Caribe: Salud Uninorte. 2004 Jul-Dic; 19: [14-24]. [Internet] Disponible en: http:// bit.ly/tEtift [consulta 01 de febrero de 2011]

16 Catácora L, Villanueva R. Conocimientos sobre VIH/SIDA y prácticas sexuales de escolares de Tacna.
Rev Peru Med Exp Salud Pública 2007; 24(3):240-3 [Internet]. Disponible en: http://bit.ly/rKB98D [consulta 01 de febrero de 2011].

17 Rojas S. Métodos para la investigación social. Plaza y Valdés, México 1992.

18 Cortes A, García R, Fullerat A. y Fuentes $\mathrm{A}$. Instrumento de trabajo para el estudio de las enfermedades de transmisión sexual y VIH/ SIDA en adolescentes. Rev Cubana Med Trop. 52(1):48-54. Cuba 2000 [Internet]; Disponible en: http:// bit.ly/rPwkF8. [consulta 01 de febrero de 2011]

19 Diario Oficial de la Federación. Reglamento de la Ley General de Salud en Materia de Investigación para la Salud. México 1984 [internet]. Disponible en: http://bit.ly/ vRUP69. [consulta 20 de enero de 2011]. 